03

\title{
О рассеянии света двухслойными эллипсоидами с несофокусными границами
}

\author{
(C) В.Б. Ильин ${ }^{1,2,3}$, В.Г. Фарафонов ${ }^{1}$, М.С. Прокопьева ${ }^{2}$, В.И. Устимов ${ }^{1}$ \\ ${ }^{1}$ Государственный университет аэрокосмического приборостроения, \\ 190000 Санкт-Петербург, Россия \\ ${ }^{2}$ Санкт-Петербургский государственный университет, \\ 199034 Санкт-Петербург, Россия \\ ${ }^{3}$ Главная (Пулковская) астрономическая обсерватория РАН, \\ 196140 Санкт-Петербург, Россия \\ e-mail: far@aanet.ru
}

Поступила в редакцию 23.03.2020 г.

В окончательной редакции 23.03.2020 г.

Принята к публикации 15.04.2020 г.

\begin{abstract}
Рассмотрены новые подходы к расчету оптических свойств малых несферических слоистых частиц, границами слоев которых являются несофокусные концентрические соосные эллипсоиды. Предложено и обосновано простое аналитическое приближение в случае, когда размер подобных частиц много меньше длины волны падающего излучения. Найдено, что приближение дает результаты с погрешностью $\sim 3 \cdot 10^{-3}$ при дифракционном параметре $x<0.1$. Обоснован подход к расчету оптических свойств несофокусных эллипсоидов, состоящий в замене несофокусных границ софокусными при сохранении объемов слоев. Показано, что для диэлектрических частиц такой подход имеет погрешность несколько процентов при $x<1$ и порядка $20 \%$ при $x<5$. Отмечено, что оба приближения легко распространяются на случай многослойных несофокусных эллипсоидов.
\end{abstract}

Ключевые слова: рассеяние света, слоистые частицы, приближение Релея.

DOI: $10.21883 /$ OS.2020.08.49712.112-20

\section{Введение}

Рассеяние света неоднородными несферическими частицами, представляет большой интерес для различных областей науки: от биофизики и экологии до астрофизики [1-3]. Часто, особенно в технических приложениях, неоднородность частиц является следствием различных покрытий их поверхности, и частицы называются слоистыми.

Взаимодействие излучения с любыми неоднородными частицами может быть рассчитано весьма затратным методом DDA (discrete dipole approximation) или сходными с ним [2]. Для слоистых частиц наряду с этими подходами может быть использован более эффективный метод ЕВСМ (extended boundary condition method [4]), который в этом случае, однако, имеет принципиальные ограничения [5].

При рассмотрении частиц, имеющих сфероидальную форму поверхности, применяют более адекватный метод SVM (separation of variables method) с соответствующим сфероидальным базисом [6]. Для слоистых частиц этот метод применим, только если границами слоев являются софокусные концентрические соосные сфероиды (в отличие от ЕВСМ других принципиальных ограничений здесь нет [7]). Для подобных (включая также эллипсоидальные) частиц, малых по сравнению с длиной волны падающего излучения, известно сравнительно простое релеевское приближение $[1,8]$.
Для несколько более сложных частиц, когда границами слоев являются несофокусные эллипсоиды, ни метод SVM, ни релеевское приближение пока не разработаны. В частном случае несофокусных сфероидов точное, но не очень эффективное, решение было предложено в [9]. Неудачным оказалось и приближение, развитое нами для подобных частиц при их размере, малом в сравнении с длиной волны [10].

Недавно было получено точное решение проблемы рассеяния света слоистыми несофокусными сфероидами в электростатическом пределе [11]. Решение основывается на новых соотношениях между сфероидальными функциями, определенными в различных системах координат [12]. Поля раскладываются по сфероидальным волновым функциям, связанным с разными (несофокусными) сфероидальными поверхностями слоев, и разложения полей согласовываются с помощью полученных соотношений между такими функциями. Расчеты показали, что для малых диэлектрических сфероидов первый ненулевой член подобных разложений является главным и аппроксимирует точное решение с погрешностью порядка $10^{-3}$ [13]. Важным является также то, что такое приближение совпадает с аналитическим приближением Релея для софокусных сфероидов той же формы и с тем же относительным объемом слоев, в котором формфакторы софокусных поверхностей заменены на формфакторы несофокусных. 
В данной работе мы применяем метод DDA для расчетов рассеяния света слоистыми эллипсоидами с целью подтвердить предположение о том, что по аналогии со сфероидами релеевское приближение для эллипсоидов с софокусными границами слоев при соответствующем изменении формфакторов является главным членом решения задачи для эллипсоидов с несофокусными границами и соответственно для частиц, малых по сравнению с длиной волны, дает результаты с погрешностью порядка $0.1 \%$. Кроме этого, рассматривается приближенный подход к частицам большего размера, состоящий в замене слоев с несофокусными границами на слои того же объема, но с софокусными границами, и обсуждается вопрос о том, почему и в какой степени подобный подход может быть приемлемой аппроксимацией. В разд. 1 показано, как формулы релеевского приближения для софокусных сфероидов появляются в решении электростатической задачи для несофокусных сфероидов, что является основой рассматриваемых аппроксимаций. В разд. 2.1 проводится сравнение приближения софокусных эллипсоидов с модифицированными формфакторами с численным решением задачи рассеяния света различными несофокусными эллипсоидами очень малого размера и определяются условия, при которых приближение является главным членом решения электростатической задачи и аппроксимирует результаты с высокой точностью. В разд. 2.2 выполняется сравнение оптических свойств сходных несофокусных и софокусных эллипсоидов, имеющих размер, сравнимый с длиной волны, и рассматривается погрешность замены одних другими. В Приложении обсуждается точность используемого метода DDA при подобных численных расчетах.

\section{1. Основные соотношения}

Рассмотрим решение проблемы рассеяния света двухслойной частицей с соосными концентрическими несофокусными сфероидальными границами слоев в электростатическом пределе и связь этого решения с приближением Релея для эллипсоидов с софокусными границами.

\section{1. Точное решение}

В электростатическом пределе все поля удовлетворяют уравнению $\Delta \mathbf{E}(\mathbf{r})=0$. Вводя скалярные потенциалы $\mathbf{E}^{(j)}=-\nabla \Phi^{(j)}$ для каждой среды $(j=1$ соответствует среде, окружающей частицу, а $j=3$ - ядру частицы), получаем уравнения для потенциалов

$$
\Delta \Phi^{(j)}(\mathbf{r})=0,
$$

которые должны быть дополнены обычными граничными условиями на границах сред [4]:

$$
\Phi^{(j)}=\Phi^{(j+1)}, \quad \frac{\partial \Phi^{(j)}}{\partial n_{j}}=\varepsilon_{j+1} \frac{\partial \Phi^{(j+1)}}{\partial n_{j}} \text { при } \mathbf{r} \in S_{j},
$$

где поверхность $S_{j}$ разделяет $j$-ю и $(j+1)$-ю среды, $n_{j}-$ внешняя нормаль к поверхности $S_{j}$, $\varepsilon_{j+1}=\epsilon_{j+1} / \epsilon_{j}-$ относительная диэлектрическая проницаемость $(j+1)$-й среды, а $\epsilon_{j}-$ проницаемость $j$-й среды.

Потенциалы представляются суммой $\Phi^{(j)}=\Phi_{1}^{(j)}+$ $+\Phi_{2}^{(j)}$, где величины с индексами 1 и 2 соответствуют регулярной и нерегулярной составляющим полей и раскладываются в соответствующие ряды по сфероидальным гармоникам $\Psi_{m l}^{(i)}(\xi, \eta, \varphi)$, где $i=1,3$ [14]. В частности, потенциалы падающего $\Phi_{1}^{(1)}$ и рассеянного $\Phi_{2}^{(1)}$ полей раскладываются по функциям, определенным для сфероидальной системы $\left(\xi_{1}, \eta_{1}, \varphi\right)$, связанной с поверхностью частицы,

$$
\begin{aligned}
& \Phi_{1}^{(1)}(\mathbf{r})=\sum_{m=0}^{\infty} \sum_{l=m}^{\infty} a_{m l}^{(1)} \Psi_{m l}^{(1)}\left(\xi_{1}, \eta_{1}, \varphi\right), \\
& \Phi_{2}^{(1)}(\mathbf{r})=\sum_{m=0}^{\infty} \sum_{l=m}^{\infty} b_{m l}^{(1)} \Psi_{m l}^{(3)}\left(\xi_{1}, \eta_{1}, \varphi\right) .
\end{aligned}
$$

Потенциалы в оболочке представляются в этой же системе как

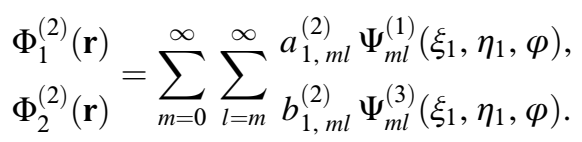

В сфероидальной системе $\left(\xi_{2}, \eta_{2}, \varphi\right)$, связанной с внутренней поверхностью оболочки, те же потенциалы имеют вид

$$
\begin{aligned}
& \Phi_{1}^{(2)}(\mathbf{r}) \\
& \Phi_{2}^{(2)}(\mathbf{r})
\end{aligned}=\sum_{m=0}^{\infty} \sum_{l=m}^{\infty} a_{2, m l}^{(2)} \Psi_{m l}^{(1)}\left(\xi_{2}, \eta_{2}, \varphi\right),
$$

Потенциал регулярного поля в ядре двухслойной частицы есть

$$
\Phi_{1}^{(3)}(\mathbf{r})=\sum_{m=0}^{\infty} \sum_{l=m}^{\infty} a_{m l}^{(3)} \Psi_{m l}^{(1)}\left(\xi_{2}, \eta_{2}, \varphi\right) .
$$

Стандартным образом, подставляя разложения потенциалов (3)-(7) в граничные условия (2) для каждой из границ оболочки и используя линейную независимость сфероидальных функций [4], получим бесконечные линейные алгебраические системы относительно коэффициентов разложения полей (неизвестными являются коэффициенты разложения потенциалов рассеянного поля $b_{m l}^{(1)}$ и внутренних полей $\left.a_{1, m n}^{(2)}, b_{1, m n}^{(2)}, a_{2, m n}^{(2)}, b_{2, m n}^{(2)}, a_{m n}^{(3)}\right)$

$$
\left\{\begin{array} { l } 
{ \mathbf { a } ^ { ( 1 ) } = A _ { 3 1 } ^ { ( 1 ) } \mathbf { a } _ { 1 } ^ { ( 2 ) } + A _ { 3 3 } ^ { ( 1 ) } \mathbf { b } _ { 1 } ^ { ( 2 ) } , } \\
{ \mathbf { b } ^ { ( 1 ) } = A _ { 1 1 } ^ { ( 1 ) } \mathbf { a } _ { 1 } ^ { ( 2 ) } + A _ { 1 3 } ^ { ( 1 ) } \mathbf { b } _ { 1 } ^ { ( 2 ) } , }
\end{array} \quad \left\{\begin{array}{l}
\mathbf{a}_{2}^{(2)}=A_{31}^{(2)} \mathbf{a}^{(3)}, \\
\mathbf{b}_{2}^{(2)}=A_{11}^{(2)} \mathbf{a}^{(3)},
\end{array}\right.\right.
$$

где использованы векторы коэффициентов

$$
\begin{gathered}
\mathbf{a}_{i}^{(j)}=\left\{a_{i, m l}^{(j)}\right\}_{l=m}^{\infty}, \mathbf{b}_{i}^{(j)}=\left\{b_{i, m l}^{(j)}\right\}_{l=m}^{\infty}, \text { матрицы } \\
A_{31}^{(j)}=\left\{\delta_{l}^{n}+\left(\varepsilon_{j+1}-1\right) L_{n l, m}^{j, 31}\right\}_{n, l=m}^{\infty},
\end{gathered}
$$




$$
\begin{gathered}
A_{33}^{(j)}=\left\{\left(\varepsilon_{j+1}-1\right) L_{n l, m}^{j, 33}\right\}_{n, l=m}^{\infty}, \\
A_{11}^{(j)}=\left\{-\left(\varepsilon_{j+1}-1\right) L_{n l, m}^{j, 11}\right\}_{n, l=m}^{\infty}, \\
A_{13}^{(j)}=\left\{\delta_{l}^{n}-\left(\varepsilon_{j+1}-1\right) L_{n l, m}^{j, 13}\right\}_{n, l=m}^{\infty}
\end{gathered}
$$

и интегралы $(i, k=1,3)$

$$
L_{n l, m}^{j, k i}=\int_{S_{j}} \Psi_{m n}^{(k)}(\mathbf{r}) \frac{\partial \Psi_{m l}^{(i)}(\mathbf{r})}{\partial n_{j}} \mathrm{~d} s .
$$

Коэффициенты разложений (5) и (6) одних и тех же потенциалов связаны соотношениями между сфероидальными функциями, которые для соосных, концентрических сфероидальных систем с фокусными расстояниями $d_{1}$ и $d_{2}$ имеют вид [12]

$$
\Psi_{m n}^{(i)}\left(\xi_{1}, \eta_{1}, \varphi\right)=\sum_{s=m}^{n}{ }^{\prime} \delta_{n s}^{(i)} \Psi_{m s}^{(i)}\left(\xi_{2}, \eta_{2}, \varphi\right),
$$

где, например,

$$
\begin{gathered}
\delta_{n s}^{(1)}=\left[\left(\frac{d_{1}}{2}\right)^{n} N_{m n}\right] \tilde{\delta}_{n s}^{(1)}\left[\left(\frac{d_{2}}{2}\right)^{s} N_{m s}\right]^{-1}, \\
\tilde{\delta}_{n s}^{(1)}=\frac{2 n+1}{2} \sum_{l=s}^{n} \frac{2(-1)^{\frac{n-l}{2}}(n+l-1) ! !}{(n-l) !(l-s) ! !(l+s+1) ! !}\left(\frac{d_{2}}{d_{1}}\right)^{l} .
\end{gathered}
$$

Здесь $N_{m s}$ - нормализующий множитель для функций Лежандра $P_{l}^{m}(\eta)$ и штрих означает суммирование только по четным или нечетным индексам в зависимости от четности $m$. Аналогично выглядят соотношения для $\delta_{n s}^{(3)}[12]$.

Применяя соотношения (14), раскладываем сфероидальную гармонику $\Psi_{m l}^{(1)}\left(\xi_{2}, \eta_{2}, \varphi\right)$ в разложении (6) в ряд по функциям $\Psi_{m l}^{(1)}\left(\xi_{1}, \eta_{1}, \varphi\right)$ и вследствие линейной независимости этих функций получаем

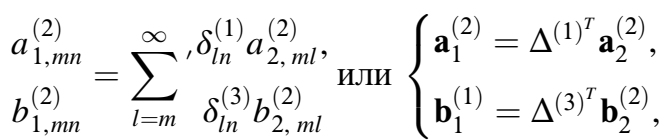

где $\Delta^{(i)}=\left\{\delta_{n s}^{(i)}\right\}_{n, s=m}^{\infty}$, а $T$ означает транспонирование.

Из уравнений (8), (17) получаем окончательную систему

$$
\left\{\begin{array}{l}
\mathbf{a}^{(1)}=A_{1} \mathbf{a}^{(3)}, \\
\mathbf{b}^{(1)}=A_{2} \mathbf{a}^{(3)},
\end{array}\right.
$$

где $A_{1}, A_{2}$ удовлетворяют соотношению

$$
\left(\begin{array}{l}
A_{1} \\
A_{2}
\end{array}\right)=\left(\begin{array}{cc}
A_{31}^{(1)} & A_{33}^{(1)} \\
A_{11}^{(1)} & A_{13}^{(1)}
\end{array}\right)\left(\begin{array}{cc}
\Delta^{(1) T} & 0 \\
0 & \Delta^{(3) T}
\end{array}\right)\left(\begin{array}{c}
A_{31}^{(2)} \\
A_{11}^{(2)}
\end{array}\right) .
$$

Исключая $\mathbf{a}^{(3)}$ из системы (18), получаем решение линейную зависимость коэффициентов разложения потенциала рассеянного поля $\mathbf{b}^{(1)}$ от аналогичных коэффициентов падающего поля $\mathbf{a}^{(1)}$ :

$$
\mathbf{b}^{(1)}=T \mathbf{a}^{(1)}, \text { где } T=A_{2} A_{1}^{-1} .
$$

Для частиц, малых в сравнении с длиной волны, оптические свойства удобно описывать с использованием поляризуемости [1]. В нашем случае поляризуемость определяется первым членом $T$-матрицы $\alpha=T_{11}$ (см. подробнее [11]), и, например, сечения ослабления и рассеяния соответственно равны $C_{\mathrm{ext}}=k \operatorname{Im} \alpha$ и $C_{\mathrm{sca}}=k^{4}|\alpha|^{2} / 6 \pi$, где $k-$ волновое число.

\section{2. Приближенные решения}

Полученное точное решение электростатической задачи позволяет обосновать несколько приближений, применимых для упрощенного расчета рассеяния света несофокусными эллипсоидами.

Рассмотрим первые элементы матриц $\Delta^{(i)}[13]$ :

$$
\Delta^{(1)}=\left(\begin{array}{cccc}
1 & 0 & 0 & 0 \\
0 & 1 & 0 & 0 \\
\Delta_{1} & 0 & 1 & 0 \\
0 & \Delta_{2} & 0 & 1
\end{array}\right), \Delta^{(3)}=\left(\begin{array}{cccc}
1 & 0 & -\Delta_{1} & 0 \\
0 & 1 & 0 & -\Delta_{2} \\
0 & 0 & 1 & 0 \\
0 & 0 & 0 & 1
\end{array}\right),
$$

где для сплюснутых сфероидальных координат $\Delta_{1}=\sqrt{21} \Delta_{d} / 8, \quad \Delta_{2}=\sqrt{45} \Delta_{d} / 8 \quad$ и $\quad \Delta_{d}=d_{1}^{2}-d_{2}^{2}, \quad$ а для вытянутых $-d$ должно быть заменено на $-i d$ (см. подробнее [13]).

Простая структура матриц $\Delta^{(i)}$ имеет важное следствие. Рассмотрим представление потенциалов в виде разложений (3)-(7), включающих только первые два члена, причем только один из них не равен нулю. Матрицы $\Delta^{(i)}$, усеченные до размера $2 \times 2$, являются единичными, и система (18), (19) становится сходной с системой для софокусных сфероидов.

Элементы матриц $A_{i k}^{(j)}$ содержат относительные проницаемости $\varepsilon_{j+1}$ и интегралы $L^{j, i k}$. В работе [11] показано, что в электростатическом пределе эти интегралы при $m=1$ равны $L_{11,1}^{j, 11}=\tilde{V}_{j}, L_{11,1}^{j, 13}=L^{j}-1, L_{11,1}^{j, 31}=L^{j}$, $L_{11,1}^{j, 33}=L^{j}\left(L^{j}-1\right) / \tilde{V}_{j}$ (аналогично для $m=0$ [8]). Здесь $\tilde{V}_{j}=3 V_{j} / 4 \pi, V_{j}-$ объем внутри поверхности $S_{j}$, $L^{j}=\int F^{-1}\left(q, a_{j}, b_{j}, c_{j}\right) \mathrm{d} q-$ формфактор этой поверхности [1]. Например, для оси $z$ фактор $L_{z}^{j}$ включает $F_{z}\left(q, a_{j}, b_{j}, c_{j}\right)=\sqrt{\left(a_{j}^{2}+q\right)\left(b_{j}^{2}+q\right)\left(c_{j}^{2}+q\right)^{3}}$. Для сфероидов интегралы $L^{j}$ берутся явно [1].

С учетом этого из равенства поляризуемости $\alpha$ первому элементу $T$-матрицы (20) получаем поляризуемость несофокусного сфероида в направлении оси $z$, совпада- 
ющей с осью симметрии частицы:

$$
\alpha_{z}=V_{1} \frac{\left(\varepsilon_{2}-1\right)\left[1+\left(\varepsilon_{3}-1\right)\left(L_{z}^{(2)}-f L_{z}^{(1)}\right)\right]}{+f \varepsilon_{2}\left(\varepsilon_{3}-1\right)} d
$$

где $L_{z}^{(2)}$ и $L_{z}^{(1)}$ - формфакторы поверхности ядра и частицы, $f=V_{2} / V_{1}$ - относительный объем ядра. Выражения для поляризуемости вдоль других осей аналогичны [13].

Соотношение (22) совпадает с выражением для поляризуемости эллипсоида с софокусными поверхностями ядра и оболочки [1]. Единственное отличие состоит в том, что для софокусных эллипсоидов полуоси ядра $\left(a_{2}, b_{2}, c_{2}\right)$ и оболочки $\left(a_{1}, b_{1}, c_{1}\right)$ и соответственно факторы $L^{(1)}$ и $L^{(2)}$ однозначно связаны с отношением объемов ядра и частицы $V_{2} / V_{1}[15]$, тогда как в формуле (22) для несофокусных сфероидов такой связи нет. Можно предположить, что для несофокусных эллипсоидов также справедливо соотношение (22), в котором все интегралы $L^{j}$ нужно находить численно, интегрируя $F^{-1}\left(q, a_{j}, b_{j}, c_{j}\right)$ с учетом величин полуосей каждой эллипсоидальной поверхности.

Важной особенностью формулы (22) является то, что, как показали численные тесты [13], первый ненулевой член разложения потенциалов, дающий это соотношение, является главным членом, представляющим решение электростатической задачи для несофокусных сфероидов с относительно малой погрешностью порядка $10^{-3}$. Мы назвали такое приближение квазисофокусным, поскольку соотношение для поляризуемости (22), которое лежит в основе этого приближения, по форме соответствует софокусному случаю и отличается от него лишь в одной указанной детали.

Высокая точность квазисофокусного приближения позволяет предположить, что замена ядра (оболочки) несофокусного эллипсоида софокусным ядром (оболочкой) того же объема может тоже быть сравнительно неплохим приближением. Мы назвали такое приближение софокусным, поскольку здесь „софокусное“ соотношение (22) применяется без модификаций. Идея подхода может быть полезна при рассмотрении оптических свойств несофокусных эллипсоидов, размер которых превосходит длину волны. В частности, оптические параметры таких несофокусных сфероидов могут быть приближенно определены в рамках данного подхода с использованием метода SVM со сфероидальным базисом, развитого, например, в [15].

Заметим, что оба приближения, определенные выше для двухслойных частиц, легко обобщаются на случай большего числа слоев, используя формулы релеевского приближения для софокусных эллипсоидов, приведенные в [8].

\section{2. Результаты численных расчетов}

\section{1. Аппроксимации для малых несофокусных эллипсоидов}

Рассмотрим, с какой точностью квазисофокусное (QCFA) и софокусное (CFA) приближения описывают оптические свойства двухслойных эллипсоидов, малых по сравнению с длиной волны падающего излучения. Заметим, что поскольку соотношения QCFA являются основным членом решения задачи рассеяния сфероидом в электростатическом пределе, то следует ожидать, что QCFA будет иметь высокую точность при малых дифракционных параметрах. Структура приближения CFA весьма близка к структуре QCFA, и поэтому возможно, что в этой области точность CFA также окажется удовлетворительной.

Применяя метод DDA (использовалась программа ADDA [16]) и приближения QCFA и CFA, мы выполнили расчеты сечений рассеяния $C_{\text {sca }}$ для двухслойных несофокусных эллипсоидов при разных значениях параметров. Считалось, что излучение распространяется вдоль средней полуоси частицы (ось $y$ ). Рассматривались частицы с подобными границами слоев, т.е. отношения полуосей поверхности частицы и ядра были равными: $a_{1} / a_{2}=b_{1} / b_{2}=c_{1} / c_{2}$. Если не указано иное, то отношение полуосей ядра и оболочки одинаковы: $a_{1}: b_{1}: c_{1}=a_{2}: b_{2}: c_{2}=10: 3: 1$, относительный объем ядра $V_{2} / V_{1}=0.421875$, показатели преломления ядра и оболочки $m_{2}=1.7+k_{2} i, m_{1}=1.3+k_{1} i$, дифракционный параметр $x_{1}=2 \pi r_{\text {eff }} / \lambda=0.001$, где $r_{\text {eff }}=\left(3 V_{1} / 4 \pi\right)^{1 / 3}-$ радиус шара, объем которого равен объему эллипсоида, $\lambda$ - длина волны излучения.

В табл. 1 приведены некоторые полученные нами результаты. В отсутствие других возможностей результаты, найденные методом DDA, использованы как базовые. Точность примененного метода DDA при решении подобных задач рассмотрена в Приложении, основной вывод которого состоит в том, что погрешность метода составляет в нашем случае порядка $3 \cdot 10^{-3}$.

Табл. 1 показывает, что относительная разность сечений, полученных методом DDA и в приближении QCFA, равна $(1-5) \cdot 10^{-3}$, т. е., скорее всего, определяется точностью, с которой в методе DDA двухслойная частица с гладкими границами описывается с помощью всего лишь миллиона диполей, расположенных на кубической решетке. Можно сделать вывод, что приближение QCFA дает сечения несофокусных эллипсоидов в электростатическом пределе (и для дифракционных параметров $x_{1}<0.1$, как видно из табл. 1) с погрешностью не более примерно $3 \cdot 10^{-3}$. Вывод справедлив для диэлектрических частиц, имеющих разные объемы ядра и оболочки и разные отношения полуосей поверхностей. Это хорошо согласуется с результатами решения электростатической задачи для слоистых сфероидов с несофокусными границами в [13]. Табл. 1 также показывает, что приближение CFA (по сути замена несо- 
Таблица 1. Сечения рассеяния $C_{\text {sca }}$, полученные методом DDA и в рамках приближения QCFA для двухслойных эллипсоидов с подобными поверхностями слоев, а также погрешности приближений QCFA и CFA

\begin{tabular}{c|c|c|c|c|c|c|c|c}
\hline \multirow{2}{*}{ Параметры } & \multicolumn{2}{|c|}{ DDA } & \multicolumn{2}{c|}{ QCFA } & \multicolumn{2}{c|}{$\mid$ QCFA-DDA $/$ DDA } & \multicolumn{2}{c}{$\mid$ CFA-DDA $/$ DDA } \\
\cline { 2 - 9 } & $C_{\text {sca } x}$ & $C_{\text {sca }, z}$ & $C_{\text {sca }, x}$ & $C_{\text {sca }, z}$ & $C_{\text {sca }, x}$ & $C_{\text {sca }, z}$ & $C_{\text {sca }, x}$ & $C_{\text {sca }, z}$ \\
\hline$a_{1}: b_{1}: c_{1}=3: 3: 1$ & $8.530 \mathrm{e}-19$ & $3.871 \mathrm{e}-19$ & $8.546 \mathrm{e}-19$ & $3.867 \mathrm{e}-19$ & $1.9 \mathrm{e}-03$ & $1.0 \mathrm{e}-03$ & $3.8 \mathrm{e}-02$ & $3.6 \mathrm{e}-02$ \\
\hline $10: 3: 1$ & $1.189 \mathrm{e}-18$ & $3.423 \mathrm{e}-19$ & $1.191 \mathrm{e}-18$ & $3.413 \mathrm{e}-19$ & $1.3 \mathrm{e}-03$ & $2.9 \mathrm{e}-03$ & $1.4 \mathrm{e}-02$ & $2.9 \mathrm{e}-02$ \\
\hline $30: 3: 1$ & $1.308 \mathrm{e}-18$ & $3.330 \mathrm{e}-19$ & $1.303 \mathrm{e}-18$ & $3.324 \mathrm{e}-19$ & $3.9 \mathrm{e}-03$ & $1.8 \mathrm{e}-03$ & $2.0 \mathrm{e}-02$ & $2.6 \mathrm{e}-02$ \\
\hline $30: 1: 1$ & $1.331 \mathrm{e}-18$ & $4.762 \mathrm{e}-19$ & $1.320 \mathrm{e}-18$ & $4.756 \mathrm{e}-19$ & $8.8 \mathrm{e}-03$ & $1.3 \mathrm{e}-03$ & $7.7 \mathrm{e}-03$ & $1.5 \mathrm{e}-03$ \\
\hline$V_{2} / V_{1}=0.244$ & $8.182 \mathrm{e}-19$ & $2.773 \mathrm{e}-19$ & $8.187 \mathrm{e}-19$ & $2.762 \mathrm{e}-19$ & $6.9 \mathrm{e}-04$ & $3.7 \mathrm{e}-03$ & $9.4 \mathrm{e}-03$ & $2.1 \mathrm{e}-02$ \\
\hline 0.422 & $1.189 \mathrm{e}-18$ & $3.423 \mathrm{e}-19$ & $1.191 \mathrm{e}-18$ & $3.413 \mathrm{e}-19$ & $1.3 \mathrm{e}-03$ & $2.9 \mathrm{e}-03$ & $1.4 \mathrm{e}-02$ & $2.9 \mathrm{e}-02$ \\
\hline 0.670 & $1.819 \mathrm{e}-18$ & $4.435 \mathrm{e}-19$ & $1.820 \mathrm{e}-18$ & $4.416 \mathrm{e}-19$ & $4.9 \mathrm{e}-04$ & $4.1 \mathrm{e}-03$ & $1.6 \mathrm{e}-02$ & $4.0 \mathrm{e}-02$ \\
\hline$k_{2} ; k_{1}=0 ; 0$ & $1.189 \mathrm{e}-18$ & $3.423 \mathrm{e}-19$ & $1.191 \mathrm{e}-18$ & $3.413 \mathrm{e}-19$ & $1.3 \mathrm{e}-03$ & $2.9 \mathrm{e}-03$ & $1.4 \mathrm{e}-02$ & $2.9 \mathrm{e}-02$ \\
\hline $0.03 ; 0.01$ & $2.165 \mathrm{e}-10$ & $6.124 \mathrm{e}-11$ & $2.169 \mathrm{e}-10$ & $6.109 \mathrm{e}-11$ & $2.2 \mathrm{e}-03$ & $2.6 \mathrm{e}-03$ & $1.7 \mathrm{e}-02$ & $3.0 \mathrm{e}-02$ \\
\hline $0.3 ; 0.1$ & $2.172 \mathrm{e}-09$ & $5.981 \mathrm{e}-10$ & $2.176 \mathrm{e}-09$ & $5.966 \mathrm{e}-10$ & $2.1 \mathrm{e}-03$ & $2.5 \mathrm{e}-03$ & $1.6 \mathrm{e}-02$ & $3.1 \mathrm{e}-02$ \\
\hline$x_{1}=0.001$ & $1.189 \mathrm{e}-18$ & $3.423 \mathrm{e}-19$ & $1.191 \mathrm{e}-18$ & $3.413 \mathrm{e}-19$ & $1.3 \mathrm{e}-03$ & $2.9 \mathrm{e}-03$ & $1.4 \mathrm{e}-02$ & $2.9 \mathrm{e}-02$ \\
\hline 0.010 & $1.189 \mathrm{e}-12$ & $3.422 \mathrm{e}-13$ & $1.191 \mathrm{e}-12$ & $3.413 \mathrm{e}-13$ & $1.3 \mathrm{e}-03$ & $2.8 \mathrm{e}-03$ & $1.4 \mathrm{e}-02$ & $2.9 \mathrm{e}-02$ \\
\hline 0.100 & $1.189 \mathrm{e}-06$ & $3.397 \mathrm{e}-07$ & $1.191 \mathrm{e}-06$ & $3.413 \mathrm{e}-07$ & $1.1 \mathrm{e}-03$ & $4.7 \mathrm{e}-03$ & $1.4 \mathrm{e}-02$ & $2.2 \mathrm{e}-02$ \\
\hline
\end{tabular}

фокусного ядра софокусным) аппроксимирует сечения с погрешностью порядка $3 \cdot 10^{-2}$. Некоторое исключение составляют сильно вытянутые сфероиды с отношением полуосей $a_{1} / b_{1}=30$, когда точность приближения CFA, возможно, возрастает.

\section{2. Аппроксимация для несофокусных эллипсоидов, сравнимых с длиной волны}

По сути наше приближение QCFA является приближением Релея для несофокусных эллипсоидов. Несмотря на относительно высокую точность для малых значений дифракционного параметра $x_{1}$, с его ростом погрешность QCFA быстро возрастает до нескольких процентов при $x_{1} \sim 0.5$. Также быстро убывает точность приближение CFA. Однако подход, использованный в CFA и заключающийся в замене несофокусного ядра (или оболочки) софокусным, имеет бо́льшую область применимости.

На рисунке приведены сечения рассеяния света двухслойными эллипсоидами с формой ядра как подобной форме частицы, так и софокусной ей. Отношение полуосей поверхности частицы $a_{1}: b_{1}: c_{1}=10: 3: 1$, относительный объем ядра $V_{2} / V_{1}=0.421875$, показатели преломления ядра и оболочки $m_{2}=1.7, m_{1}=1.3$. В обоих случаях применялся метод DDA. Из рисунка видно, что сечение рассеяния софокусного эллипсоида аппроксимируют сечения несофокусного того же объема с погрешностью менее $20 \%$ в широком диапазоне значений дифракционного параметра: примерно для $x_{1}<5$.
Аппроксимация с погрешостью нескольких процентов имеет место при $x_{1}<0.5$. Как показывает правая панель рисунка, эти выводы справедливы для почти любых диэлектрических материалов.

Замену несофокусного эллипсоида софокусным можно делать по-разному, например, изменяя форму либо ядра, либо оболочки; в последнем случае форма ядра сохраняется. Вероятно, выбор способа зависит от вклада ядра и оболочки в сечение поглощения в релеевском приближении, который определяется объемом слоев и мнимой частью их поляризуемости $\operatorname{Im}\{\alpha\}$.

\section{Заключение}

Рассмотрено решение проблемы рассеяния света двухслойным сфероидом с несофокусными границами слоев в электростатическом пределе, и показаны теоретические основания двух новых приближений для эллипсоидов с несофокусными границами.

Приближение, названное квазисофокусным, представляет собой релеевское приближение для софокусного эллипсоида с таким же объемом ядра и оболочки как у несофокусного. Однако в простые выражения этого приближения подставляются формфакторы, рассчитанные для несофокусных границ. Численно показано, что по аналогии со случаем слоистых сфероидов квазисофокусное приближение представляет собой главный член решения задачи для несофокусных эллипсоидов в электростатическом пределе и соответственно при ма- 

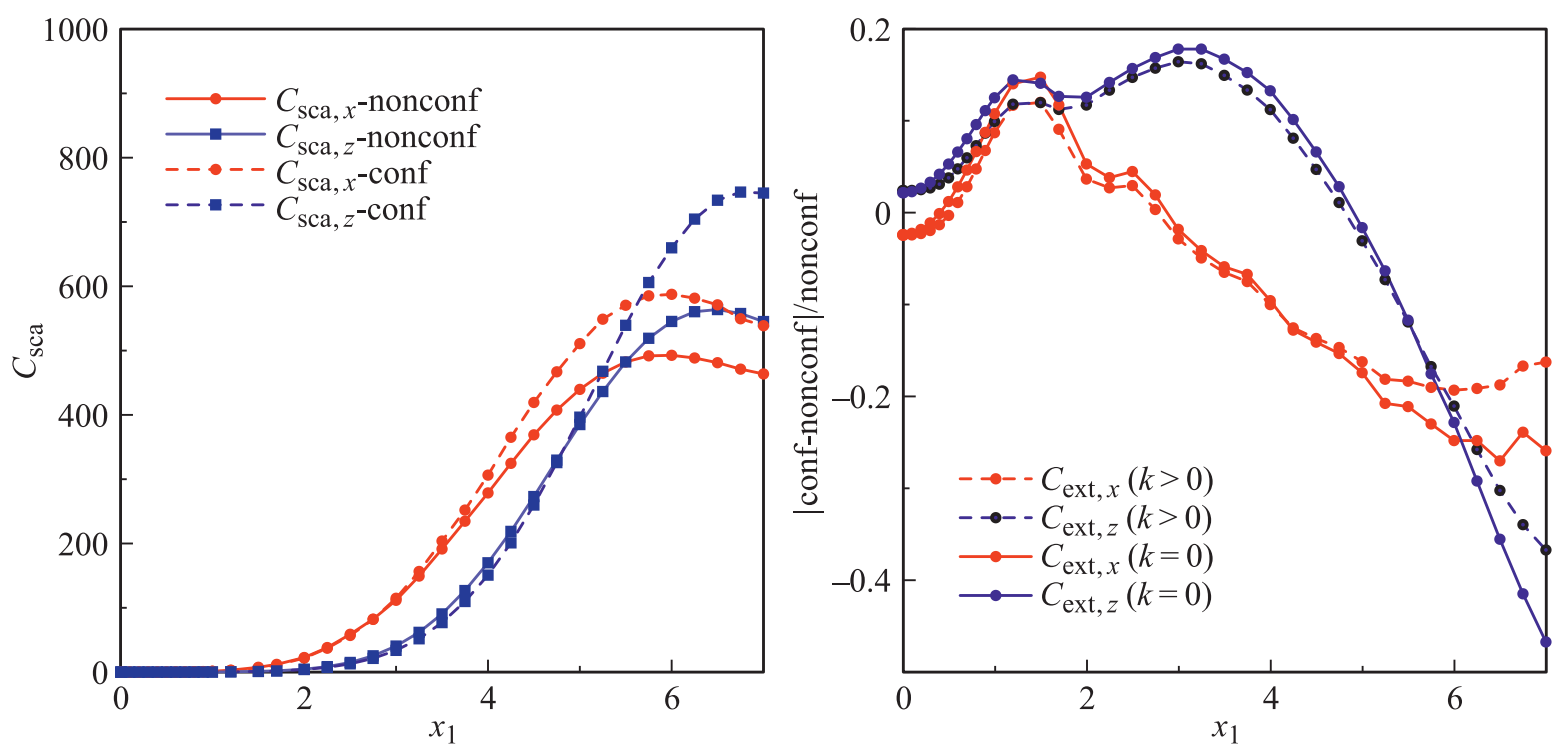

Левая панель: сечения рассеяния $C_{\text {sca, }}$ рассчитанные методом DDA для двухслойного несофокусного (подобного) эллипсоида (случай nonconf) и сходного эллипсоида с софокусным ядром (conf) при разном дифракционном параметре $x_{1}$; отношение полуосей частиц $a_{1}: b_{1}: c_{1}=10: 3: 1$, относительный объем ядра $V_{2} / V_{1}=0.421875$, показатели преломления ядра и оболочки $m_{2}=1.7, m_{1}=1.3$. Правая панель: относительная разность сечений поглощения $C_{\text {ext }}$, рассчитанных для вышеуказанных эллипсоидов при $m_{2}=1.7, m_{1}=1.3$ (сплошные линии) и $m_{2}=1.7+0.03 i, m_{1}=1.3+0.01 i$ (штриховые линии).

лом дифракционном параметре $(x<0.1)$ имеет малую погрешность, например около $(1-5) \cdot 10^{-3}$ при расчете сечений для диэлектрических частиц.

Другое приближение, названное софокусным, представляет собой замену несофокусного ядра (или оболочки) софокусным. При малых дифракционных параметрах $(x<0.3)$ можно использовать соответствующее релеевское приближение, и тогда погрешность такого подхода порядка $3 \cdot 10^{-2}$. При бо́льших значениях параметра подход аппроксимирует сечения с погрешностью нескольких процентов при $x<1$ и с погрешностью порядка $20 \%$ при $x<5$. Заметим, что основанием этого подхода является его близость в электростатическом пределе к главному члену точного решения для несофокусных эллипсоидов и неожиданно быстрое убывание остальных его членов.

Приближения сформулированы для двухслойного эллипсоида, но легко распространяются на случай многослойных.

\section{Благодарности}

Авторы благодарны М. Юркину за возможность использовать программу ADDA.

\section{Финансирование работы}

Работа была поддержана в 2020 г. грантом ГУАП и грантом РФФИ 18-52-52006.

\section{Конфликт интересов}

Авторы заявляют, что у них нет конфликта интересов.

\section{Приложение}

Метод DDA, примененный нами для моделирования оптических свойств слоистых частиц, является точным только в пределе, когда число диполей, использованных для представления частиц, стремится к бесконечности. Компьютеры, применявшиеся нами, позволяли проводить вычисления лишь для примерно $10^{6}$ диполей, что ограничивало точность численных расчетов.

Оценки погрешности результатов, полученных методом DDA, были сделаны нами двумя способами. Вопервых, использовался тот факт, что для непоглощающих частиц сечения поглощения и рассеяния должны быть равны. Поскольку эти сечения рассчитываются разным образом, их сравнение позволяет судить о точности метода. Во-вторых, в частном случае софокусных сфероидов сравнивались результаты, полученные методом DDA (использовалась программа ADDA [16]) и хорошо проверенным точным методом SVM со сфероидальным базисом (применялась программа, описанная в [15]).

Тестовые расчеты были проведены для двухслойных вытянутых сфероидов с софокусными поверхностями оболочки. Отношение полуосей поверхности частицы $a_{1} / b_{1}=1.5$, относительный объем ядра $V_{2} / V_{1}=0.50624$, дифракционный параметр $x_{1}=0.001$. Рассматривались разные пары показателей преломления ядра $m_{2}$ и оболочки $m_{1}$, углы наклона оси симметрии частицы к волновому вектору $\alpha$ и направления поляризации падающего излучения. Некоторые результаты приведены в табл. 2.

Второй столбец табл. 2 содержит относительную разность сечений поглощения и рассеяния, рассчитанных 
Таблица 2. Относительная разность сечений поглощения и рассеяния, полученных методами DDA и SVM со сфероидальным базисом для двухслойных вытянутых сфероидов с софокусными поверхностями слоев

\begin{tabular}{|c|c|c|c|c|c|}
\hline \multirow{3}{*}{ Параметры } & DDA & \multicolumn{4}{|c|}{ |DDA-SVM|/SVM } \\
\hline & \multirow{2}{*}{$2 \frac{\left|C_{\text {ext }}-C_{\text {sca }}\right|}{C_{\text {ext }}+C_{\text {sca }}}$} & \multicolumn{2}{|c|}{$m_{1} / m_{2}=1.7 / 1.3$} & \multicolumn{2}{|c|}{$1.7+0.03 i / 1.3+0.01 i$} \\
\hline & & $C_{\text {ext }}$ & $C_{\text {sca }}$ & $C_{\text {ext }}$ & $C_{\text {sca }}$ \\
\hline$\alpha=0$ & $5.3 e-06$ & $7.5 \mathrm{e}-04$ & $7.6 e-04$ & $1.1 \mathrm{e}-03$ & 7.6e-04 \\
\hline$\alpha=90^{\circ}$, TЕ-мода & $5.6 \mathrm{e}-06$ & $7.5 \mathrm{e}-04$ & $6.3 \mathrm{e}-04$ & $1.1 \mathrm{e}-03$ & $9.1 \mathrm{e}-04$ \\
\hline$\alpha=90^{\circ}$, ТМ-мода & $1.7 \mathrm{e}-06$ & $7.6 \mathrm{e}-04$ & $6.3 e-04$ & $7.6 \mathrm{e}-04$ & $6.3 \mathrm{e}-04$ \\
\hline
\end{tabular}

методом DDA, $\left|C_{\text {ext }}-C_{\text {sca }}\right| /\left(C_{\text {ext }}+C_{\text {sca }}\right) / 2$. Эта разность составляет порядка $5 \cdot 10^{-6}$ и характеризует „внутреннюю“ точность метода, которая обычно примерно на 1-1.5 порядка выше реальной.

Столбцы 3-6 таблицы показывают относительную разность сечений поглощения или рассеяния, рассчитанных разными методами для одинаковых слоистых сфероидов и двух пар значений показателей преломления ядра и оболочки. Программа, основанная на методе SVM со сфероидальным базисом и примененная нами для расчета оптических свойств двухслойных софокусных сфероидов, хорошо протестирована и имеет точность более 8 значащих цифр при указанных значениях параметров. Поэтому столбцы 3-6 показывают, что в данном случае программа ADDA дает значения сечений $C_{\text {ext }}, C_{\text {sca }}$ для диэлектрических частиц с погрешностью около $10^{-3}$.

Существенное отличие этого значения от „внут-

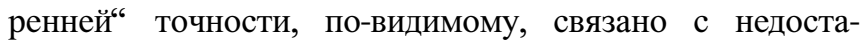
точным числом диполей для адекватной аппроксимации формы и структуры частиц. Некоторым подтверждением этому служит следующий численный тест. Был рассмотрен двухслойный софокусный эллипсоид с полуосями $a_{1}: b_{1}: c_{1}=10: 3: 1$ (вписанный в массив диполей $400 \times 120 \times 40)$, относительным объемом ядра $V_{2} / V_{1}=0.4253625$ (ядро вписано в массив $398 \times 114 \times 18)$, показателями преломления ядра и оболочки $m_{2}=1.7, \quad m_{1}=1.3$ и дифракционным параметром $x_{1}=0.001$ при падении излучения вдоль средней полуоси. Расчеты методом DDA дали $C_{\mathrm{sca}, x}=1.2168 \cdot 10^{-18}, C_{\mathrm{sca}, z}=3.3464 \cdot 10^{-19}$. Релеевское приближение для софокусного эллипсоида (CFA) с такими же параметрами и отношением полуосей ядра $a_{2}: b_{2}: c_{2}=398: 114: 18$, соответствующим дипольной DDA-модели ядра, дал $C_{\mathrm{sca}, x}=1.2134 \cdot 10^{-18}$, $C_{\mathrm{sca}, z}=3.3361 \cdot 10^{-19}$. Относительная разность DDA и CFA оказалась равной $\sim 3 \cdot 10^{-3}$, что сходно с нашей оценкой точности QCFA в табл. 1 и по порядку величины близко к относительной разности результатов, полученных методами DDA и SVM для вытянутых софокусных сфероидов и приведенных в табл. 2.

Таким образом, при использованном представлении слоистых частиц диполями погрешность, с которой метод DDA воспроизводит сечения двухслойных эллипсоидов, составляет порядка $\sim 3 \cdot 10^{-3}$.

\section{Список литературы}

[1] Борен К., Хабббмен Д. Поглощение и рассеяние света малыми частицами. М.: Мир, 1986.

[2] Mishchenko M.I., Hovenier J.W., TravisL.D. // Light Scattering by Nonspherical Particles. San Diego: Academic Press, 2000.

[3] Mishchenko M.I., Travis L.D., Lacis A.A. // Scattering, Absorption and Emission of Light by Small Particles. Cambridge: Cambridge Univ. Press, 2002.

[4] Farafonov V.G., Ilin V.B. // Light Scatt. Rev. 2006. V. 1. P. 125.

[5] Farafonov V.G., Il'in V.B., Ustimov V.I., Volkov E.V. // Adv. Math. Phys. 2017. V. 2017. N 7862462.

[6] Voshchinnikov N.V., Farafonov V.G. // Astrophys. Sp. Sci. 1993. V. 204. P. 19.

[7] Farafonov V.G. // Light Scatt. Rev. 2013. V. 8. P. 189.

[8] Farafonov V.G., Sokolovskaya M.V. // J. Math. Sci. 2013. V. 194. P. 104.

[9] Han Y.P., Zhang H.Y., Sun X. // Appl. Phys. B. 2006. V. 84. P. 485.

[10] Posselt B., Farafonov V.G., Il'in V.B., Prokopjeva M.S. // Meas. Sci. Technol. 2002. V. 13. P. 256.

[11] Фарафбонов В.Г., Устимов В.И., Ильин В.Б. // Опт. и спектр. 2019. Т. 125. С. 786.

[12] Фарафонов В.Г., Устимов В.И., Ильин В.Б. // Зап. Науч. семин. ПОМИ. 2019. Т. 483. С. 199.

[13] Фарафбонов В.Г., Устимов В.И., Ильин В.Б. // Опт. и спектр. 2019. Т. 126. С. 450.

[14] Комаров В.И., Пономарев Л.И., Славянов С.Ю. Сфероидальные и кулоновские сфероидальные функции. М.: Наука, 1976.

[15] Farafonov V.G., Voshchinnikov N.V. // Appl. Opt. 2012. V. 51. P. 1586.

[16] Yurkin M.A., Hoekstra A.G. // J. Quant. Spectrosc. Rad. Transf. 2011. V. 112. P. 2234. 